\title{
ARDS and Neurological Outcome: There is not just One Organ to Protect!
}

\author{
Chiara Robba* (1)
}

๑ 2021 Springer Science+Business Media, LLC, part of Springer Nature and Neurocritical Care Society, corrected publication 2023

Despite improvement in the knowledge on the pathophysiology and management of acute respiratory distress syndrome (ARDS), the mortality rate remains very high [1]. In addition, survivors of ARDS may experience long-term morbidity, with a large incidence of neurological sequelae, such as delirium, cognitive dysfunction, and neuropsychiatric morbidities [1].

Acute brain injury (ABI) can frequently occur after ARDS because of cross talk between the lung and the brain, which is a complex relationship involving different pathways [2]. Several pathophysiological mechanisms have been explored regarding the secondary effects of lung damage and ARDS on the brain; these include the recognition of ventilator-induced lung injury as determinant of systemic inflammation affecting distal organs, including the brain, as well as the role of mechanical ventilation in maintaining inflammation and activating cytokines cascade [2]. In addition, optimal ventilatory strategies for patients with lung injury are potentially in conflict with cerebral needs; for instance, the use of high positive end expiratory pressure could lead to reduced cerebral venous outflow and cause cerebral edema and low tidal volume, and consequent permissive hypercapnia can lead to cerebral vasodilation and increased intracranial pressure [3].

Protecting the lung while protecting the brain is considered a new target in the modern intensive care setting, but the possible implications of ARDS and lung-protective strategies on the brain are undiscovered areas of research in experimental and clinical settings [3]. This is further highlighted in the era of the coronavirus disease 2019 pandemic, considering the extremely high

\footnotetext{
*Correspondence: chiara.robba@unige.it

Department of Surgical Science and Diagnostic Integrated, Anesthesia and Intensive Care, Policlinico San Martino, IRCCS for Oncology

and Neuroscience, University of Genova, Genova, Italy
}

number of neurological complications detected in this population.

The article by Huang et al. [4] aimed to clarify this issue. The authors performed a systematic review and summarized the existing literature on ARDS-associated $\mathrm{ABI}$ in clinical studies and preclinical models to elucidate potential mechanisms of ABI in ARDS.

Thirty-eight studies were included (28 clinical studies with 1175 patients, 10 preclinical studies with 143 experimental models), encompassing 12 studies on neurocognitive outcomes ( $n=797$ patients), 3 clinical observational studies $(n=141), 13$ clinical case series/reports $(n=15)$, 9 animal studies $(n=143)$, and 1 in vitro study.

High frequency of ARDS-associated ABI was observed, ranging from 53 to $86 \%$ in observational studies. Of the reported ABIs (median age 49 years, $46 \%$ men), the most common injury was hemorrhagic stroke (25\%), followed by hypoxic ischemic brain injury (22\%), diffuse cerebral edema (11\%), and ischemic stroke (8\%). Cognitive impairment in patients with ARDS $(n=797)$ was observed in $87 \%$ cases (range $73-100 \%$ ) at discharge, 36\% (range $32-37 \%$ ) at 6 months, and 30\% (range $25-45 \%$ ) at 1 year.

Most ARDS animal studies demonstrated evidence of neuroinflammation and neuronal damage, mainly occurring within the hippocampus, as well as altered cerebral blood flow and increased intracranial pressure with use of lung-protective mechanical ventilation. Overall, paucity of data was observed regarding the prevalence of $\mathrm{ABI}$ in patients with ARDS, but the authors concluded that the main mechanisms of ARDS-associated ABI include primarily hypoxic ischemic injury from hypoxic respiratory failure, secondary injury from lung injury induced neuroinflammation, and increased intracranial pressure from ARDS lung-protective mechanical ventilation strategies.

\section{Springer}


Although this systematic review is well conducted, including a robust and strict methodology in the search strategy and selection of the studies, quality assessment, and risk of bias evaluation, several limitations have been acknowledged by the authors. These include the significant heterogeneity regarding the definition and assessment of cognitive function, the absence of a pre-ARDS basal cognitive evaluation, and the lack of standardization in the definition of the severity of ARDS and of systemic patients' conditions. However, results from this review offer the opportunity to deeply understand the pathophysiological mechanisms relying on the occurrence of neurological consequences in patients with ARDS and potential targets of treatment to prevent, reduce, or promptly treat these complications.

Neuroinflammation and neuronal apoptosis triggered by lung damage may exacerbate cerebral damage, thus suggesting that the application of a protective approach in mechanical ventilation is warranted. However, the fact that lung-protective strategies may potentially alter cerebral blood flow and function makes them a double edge sword and poses the question of whether it is safe to use them and which organ, between brain and lung, has the priority for protection.

In fact, recent consensus statements push toward the application of lung protective strategies, as they can improve outcomes even in patients with brain injury [3]; however, when these strategies are applied, higher attention should also be paid to protecting the brain with the use of multimodal neuromonitoring tools. Despite invasive methods are not recommended for patients with nonprimarily brain injury, a number of noninvasive techniques (such as electroencephalography, transcranial Doppler, near infrared spectroscopy, and pupillometry) have been recently proposed as bedside tools able to early and safely detect altered cerebral perfusion, oxygenation, and increased intracranial pressure [5]. Although not yet validated for their routine use in the general critically ill population, these tools have been applied in the neurocritical care setting as well as in the operating room, suggesting a potential role in the reduction of neurological sequelae [5]. The implementation of these tools may potentially allow the adoption of therapeutic strategies aimed to mitigate the possible detrimental cerebral effects of lung protective strategies.

In summary, results from the review from Huang et al. [4] highlight the important issue of the severity and high prevalence of neurological complications occurring in patients with ARDS. These can be consequent to the systemic release of inflammatory markers and pathways directly correlated with lung injury and mechanical ventilation itself. Protecting the lung should remain our goal as clinicians, but we should also learn to look at the cerebral needs, especially in patients with severe ARDS requiring aggressive treatment and patients at risk for neurological complications, by monitoring cerebral function. Further research is warranted to assess the role of these tools in this group of patients and validate their use, as having potentially beneficial effects on short-term and long-term outcome.

\section{Source of support}

None

\section{Conflict of interest}

The authors declared that they have no conflict of interest

\section{Publisher's Note}

Springer Nature remains neutral with regard to jurisdictional claims in published maps and institutional affiliations.

Received: 18 May 2021 Accepted: 20 May 2021

Published: 26 July 2021

References

1. Bellani G, Laffey JG, Pham T, et al. Epidemiology, patterns of care, and mortality for patients with acute respiratory distress syndrome in intensive care units in 50 countries. JAMA. 2016;315:788-800.

2. Blanch L, Quintel M. Lung-brain cross talk in the critically ill. Intensive Care Med. 2017:43:557-9.

3. Robba C, Poole D, McNett M, et al. Mechanical ventilation in patients with acute brain injury: recommendations of the European Society of Intensive Care Medicine consensus. Intensive Care Med. 2020;46(12):2397-410.

4. Huang M, Gedansky A, Hasset CE, et al. Pathophysiology of brain injury and neurological outcome in acute respiratory distress syndrome: a systematic scoping review of preclinical to clinical studies. Neurocrit Care. 2021;35:518-527. https://doi.org/10.1007/s12028-021-01309-x.

5. Vinciguerra L, Bösel J. Noninvasive neuromonitoring: current utility in subarachnoid hemorrhage, traumatic brain injury, and stroke. Neurocrit Care. 2017;27(1):122-40. 